\title{
Desempenho Criativo e suas Relações com Diferentes Medidas de Inteligência em Crianças com Dislexia do Desenvolvimento: Um Estudo Exploratório
}

\author{
Creative Performance and their Relationship with Different Measures \\ of Intelligence in Children with Developmental Dyslexia: An Exploratory Study
}

\author{
Rauni Jandé Roama Alves* \& Tatiana de Cássia Nakano \\ Pontificia Universidade Católica de Campinas, Campinas, SP, Brasil
}

\begin{abstract}
Resumo
Com o objetivo de verificar o desempenho criativo e suas relações com diferentes medidas de inteligência em uma população de crianças com Dislexia do Desenvolvimento, 13 participantes com esse diagnóstico foram avaliados, $61 \%(n=8)$ do sexo masculino e $39 \%(n=5)$ do sexo feminino, com idades entre nove e 11 anos $(M=10,92+1,03)$. Utilizaram-se os seguintes testes psicológicos: Teste de Criatividade Figural Infantil (TCFI), Escala de Inteligência Wechsler para crianças (WISC-III), Matrizes Progressivas Coloridas de Raven (MPCR) e o Desenho da Figura Humana (DFH-III). Os resultados mostraram, tanto em relação à criatividade quanto à inteligência, maior concentração da classificação "média", havendo, no instrumento de criatividade, melhor desempenho no fator emotividade. Quatro participantes apresentaram desempenho acima da média, podendo ser enquadrados no diagnóstico denominado de dupla excepcionalidade, o qual merece ser aprofundado. Na investigação das correlações entre inteligência e criatividade não foi verificado um padrão de achados (correlação alta e significativa entre o MPCR e o TCFI, $r=0,80$; não significativa para o DFH e o WISC-III com TCFI). Considerando-se a multidimensionalidade dos construtos envolvidos, novas investigações que envolvam o grupo investigado fazem-se necessárias, a fim de confirmar ou não os resultados aqui encontrados.

Palavras-chave: Dislexia, avaliação psicológica, criatividade, aptidão cognitiva.
\end{abstract}

\begin{abstract}
In order to verify the creative performance and its relationship with different measures of intelligence in a population of children with Developmental Dyslexia, 13 participants diagnosed with this framework were evaluated, $61 \%(n=8)$ were male and $39 \%(n=5)$ female, aged between nine and 11 years $(M=10.92+1.03)$, using the following psychological tests: Test of Children's Figural Creativity (TCFI), Wechsler Intelligence Scale for Children (WISC-III), Raven Colored Progressive Matrices (RCPM) and the Human Figure Drawing (HFD-III). Results showed that there was higher concentration of "average" ratings regarding creativity as well as intelligence. Also, the creativity instrument had better performance in the emotionality factor. Four participants had above average performance and can be classified into the so-called dual exceptionality diagnosis, which should be further investigated. Regarding correlations between intelligence and creativity, there was no pattern in the findings (high and significant correlation between RCPM and TCFI, $r=.80$, not significant for the HFD and the WISC-III with TCFI). Considering the multidimensionality of the constructs involved, further studies involving the investigated group are necessary in order to confirm or refute the results found here.

Keywords: Dyslexia, psychological assessment, creativity, cognitive ability.
\end{abstract}

A Dislexia do Desenvolvimento é um transtorno de aprendizagem que envolve desde dificuldades para aprender a ler até problemas para adquirir proficiência em escrita e ortografia. Sua origem é neurobiológica e as dificuldades

\footnotetext{
${ }^{*}$ Endereço para correspondência: Pontifícia Universidade Católica de Campinas, Centro de Ciências da Vida, Av. John Boyd Dunlop, s/n, Jardim Ipaussurama, Campinas, SP, Brasil 13060-904. E-mail: rauniroama@gmail.com e tatiananakano@hotmail.com
}

concentram-se principalmente no reconhecimento das palavras e nas dificuldades em soletração (Lyon, Shaywitz, \& Shaywitz, 2003), não devendo, no entanto, ser atribuída à deficiência intelectual, aspectos motivacionais, escolarização inadequada e a comprometimentos sensoriais, de acordo com critérios da American Psychiatric Association (APA, 2002) e Organização Mundial da Saúde (2008).

Raramente o meio clínico e escolar, e até mesmo o científico, buscam reconhecer e investigar a possibilidade 
de que sujeitos com desenvolvimento diverso ao esperado possam apresentar melhores habilidades cognitivas que aqueles com desenvolvimento considerado normal (Neumeister, Yssel, \& Burney, 2013). Esse padrão cognitivo pode ser encontrado na literatura sob a denominação de "dupla excepcionalidade", definida como a presença de uma alta performance, talento, habilidade ou potencial (por exemplo, em criatividade e inteligência) ocorrendo em conjunto com uma desordem médica ou psiquiátrica ou ainda incapacidade educacional (Pfeiffer, 2013).

Tal assunto tem se constituído em interesse da comunidade científica desde a década de 1970, baseado em observações realizadas em situações clínicas e de aconselhamento, pesquisa desenvolvidas em áreas específicas de talento (que acabaram por encontrar, quase que "acidentalmente" a superdotação associada a algum outro quadro), observações comportamentais e observação nas salas de aula (Kalbfleisch \& Iguchi, 2008), os quais acabaram por servir de base para o que se entende sobre a dupla-excepcionalidade. Desse modo, pode-se notar que seu estudo tem se mostrado muito mais resultado de casos encontrados na prática do que estudos teóricos, haja vista que, até o momento, nenhuma teoria específica tenha sido elaborada para sua explicação. Considerando-se ainda que, comumente a maior parte dos indivíduos portadores de alguma incapacidade ainda é reconhecida por suas dificuldades e não por seus talentos (Davis, Rimm, \& Siegle, 2011), essa área de pesquisa tem apresentado grande potencial de contribuir para a compreensão mais global dessa população especial, de modo a auxiliar na elaboração de programas de atendimento mais eficazes.

De acordo com Kalbfleisch e Iguchi (2008), em estudantes que apresentam altas habilidades / superdotação e dislexia concomitantemente pode-se observar dificuldade em habilidades verbais e de seqüenciamento, juntamente com boa performance em tarefas que requerem uso de blocos, design visual e habilidades espaciais. Especificamente para a associação entre criatividade e dislexia, pode-se citar a pesquisa realizada por Alves e Nakano (no prelo). Os autores visaram identificar a produção científica, nacional e internacional, sobre criatividade e transtornos de aprendizagem, envolvendo oito bases de dados eletrônicas: APA, Education Resources Information Center (ERIC), PubMed, Scopus, Scientific Electronic Library Online (SCIELO), Periódicos Eletrônicos de Psicologia (PEPSIC), base de teses e dissertações da Coordenação de Aperfeiçoamento de Pessoal de Nível Superior (CAPES) e Biblioteca Digital de Teses e Dissertações (BDTD), em um total de 62 trabalhos analisados, trouxe resultados que apontam para o fato de que tal quadro não tem sido investigado no país.

Para dislexia não foi encontrado nenhum trabalho nacional, já internacionalmente verificaram-se 17 produções, mas apenas cinco de estudos exploratórios, que buscaram verificar as características criativas presentes em indivíduos com esse transtorno. A partir desses resultados, os autores concluíram que a literatura tem sido marcada pela ausência de consenso sobre a questão (se, por exemplo, indivíduos com dislexia apresentariam melhores desempenhos criativos ou não quando comparados a indivíduos sem dificuldades de aprendizagem), além do número pequeno de investigações, de modo a constituir-se em um foco a ser estudado.

Dentre os estudos encontrados, com foco na criatividade e dislexia, pode-se citar a pesquisa desenvolvida por Pachalska, Bogdanowicz, Tomaszewska, Łockiewicz e Bogdanowicz (2009), que indicou que adultos com dislexia apresentavam melhor uso e habilidade de aplicações para cores e também maiores detalhes na realização de desenhos realísticos. Everatt, Steffert e Smythe (1999), verificaram que adultos com dislexia apresentaram melhor desempenho em habilidades criativas de originalidade e insight, também apresentando estilos de pensar mais inovadores. Por outro lado, grupos de crianças e adolescentes com dislexia apresentaram melhores desempenhos, de modo geral, em testes figurais.

Em relação a pesquisas realizadas especificamente com crianças, pode-se citar primeiramente a de Çorlu, Özcan e Korkmazlar (2007). Os autores verificaram maior riqueza de detalhes em tarefas de design realizadas por crianças com dislexia, quando avaliadas por um grupo de juízes especialistas na área. Em outra investigação, os mesmos autores (Çorlu, Özcan, \& Korkmazlar, 2009) concluíram que crianças com dislexia apresentaram melhor desempenho e velocidade na produção de figuras originais. Também Tafti, Hameedy e Baghal (2009) verificaram que as crianças com dislexia apresentaram melhor desempenho em originalidade figurativa quando comparadas a crianças sem esse transtorno sendo que, de maneira geral, ambos os grupos não apresentaram diferenças significativas no desempenho geral em criatividade.

Nesse sentido, alguns autores (Chakravarty, 2009; Cohn \& Neumann, 1977; J.-Y. Kim \& Ko, 2007) apontam que a base explicativa para esse melhor desempenho em criatividade estaria em condições cerebrais: especulam que o hemisfério direito seria mais desenvolvido funcionalmente em indivíduos com dislexia, principalmente o lobo parietal, sendo tal condição responsável pelo talento e por produções criativas. Já estudos em neuropsicologia apontam para uma ligação entre a criatividade figural e as habilidades visoespaciais (Károlyi \& Winner, 2004; West, 2009). Pesquisas nessa área verificaram um melhor desempenho de indivíduos com dislexia em habilidades visoespaciais quando submetidos a investigações com maior validade ecológica (Brunswick, Martin, \& Marzano, 2010; Eide \& Eide, 2011; Winner et al., 2001), o que poderia, dessa forma, explicar o melhor desempenho criativo dessa população.

Um segundo tópico importante quando se estuda criatividade, refere-se à sua relação com a inteligência (Guilford, 1967; Sternberg \& O'Hara, 1999; Torrance, 1962; Vernon, 1964). Investigações atuais seguem questionando a existência dessa relação e o nível da associação, além da sua estabilidade no tempo e possibilidade 
de generalização para populações distintas (Elisondo \& Donolo, 2010).

Nakano (2012), por meio de uma revisão da literatura, apontou a existência de três vertentes teóricas comumente discutidas entre os autores mais renomados na área: (a) a existência de relação somente a partir de certo nível de QI (usualmente 120), denominado de threshold theory; (b) aqueles que afirmam que ambos os construtos estariam altamente relacionados e ainda (c) aqueles que defendem a independência entre eles. Desse modo, os principais focos de debate seriam se tais construtos são habilidades sinônimas, diferenciadas ou sobrepostas (Wechsler, Nunes, Schelini, Ferreira, \& Pereira, 2010).

Nesse sentido, Preckel, Holling e Wiese (2006) afirmam que algumas variáveis são tomadas como explicativas para as amplas variações de correlações encontradas. Uma delas estaria relacionada com a diversidade dos instrumentos utilizados nas pesquisas, em que as correlações variariam de acordo com a forma em que a criatividade e a inteligência estão sendo medidas. Os autores apontam ainda para outras variáveis, como as diferenças metodológicas e a amostra estudada (que varia em função da idade, habilidade e nível educacional). Embora a maior parte dos estudos tenha concluído acerca da existência de alguma relação entre ambos os construtos, com a amplitude desta relação variando entre baixa e moderada, as diferenças relatadas acabam dificultando o consenso em relação a esta questão (Nakano \& Brito, 2013).

Diante do exposto, o presente estudo, de caráter exploratório, objetivou investigar o desempenho criativo de crianças com Dislexia do Desenvolvimento. Além disso, buscou-se ainda estimar as correlações entre esse desempenho e diferentes medidas de inteligência, a fim de verificar se nessa amostra encontrar-se-ia um padrão, ou se assim como apontado na literatura as correlações variariam em razão do uso de diferentes instrumentos. A partir desse objetivo visou-se, secundariamente, também discutir a possibilidade da presença da dupla-excepcionalidade nessa mesma população.

Vale esclarecer que a definição de criatividade que embasa o estudo é a elaborada por Torrance (1965), na qual a criatividade pode ser definida como um:

processo de tornar-se sensível a problemas, deficiências, lacunas, elementos ausentes ou desarmonias; identificar as dificuldades ou os elementos faltantes nas informações; formular hipóteses, fazendo adivinhações a respeito das deficiências encontradas; testar e retestar estas hipóteses, possivelmente modificá-las e retestá-las novamente. (p. 8)

Atualmente, existe apenas um instrumento para avaliar criatividade no Brasil que atenda a faixa etária do presente estudo e que seja aprovado pelo Conselho Federal de Psicologia, denominado de "Teste de Criatividade Figural Infantil" (Nakano, Wechsler, \& Primi, 2011), que, além disso, é baseado nessa perspectiva de Torrance. Para sua correlação com medidas de inteligência, foram empregados alguns dos testes mais frequentemente conhecidos e utili- zados por psicólogos no país (Noronha, Primi, \& Alchieri, 2005), e que atendesse a faixa etária do presente estudo: "Escala de Inteligência Wechsler para Crianças" (Figueiredo, 2002), "Matrizes Progressivas Coloridas de Raven" (Angelini, Alves, Custódio, Duarte, \& Duarte, 1999) e "Desenho da Figura Humana" (DFH-III; Wechsler, 2003).

\section{Método}

\section{Participantes}

Treze crianças com Dislexia do Desenvolvimento, sendo $61 \%(n=8)$ do sexo masculino e $39 \%(n=5)$ do sexo feminino, com idades entre 9 e 11 anos $(M=10,92 ; D P=1,03)$, e série escolar variando do terceiro ao sétimo ano, com maior concentração nas séries quinto $(38,5 \% ; n=5)$ e sexto ano $(30,8 \% ; n=4)$. A grande maioria pertencia à escola pública $(n=10)$. Todas as crianças foram selecionadas por conveniência no ambulatório de "Neuro-Dificuldades de Aprendizagem" do Hospital de Clínicas da Universidade Estadual de Campinas (UNICAMP). Os critérios de inclusão para a presente pesquisa foram: possuir faixa etária entre oito e 11 anos e oito meses (dada a necessidade de adequação da idade do participante com a faixa etária compreendida nos instrumentos psicológicos utilizados na pesquisa); assinatura do Termo de Consentimento Livre e Esclarecido (TCLE) pelos pais ou responsáveis, tendo-se excluído todas as crianças cujo diagnóstico não tivesse sido fechado pela equipe do ambulatório de Neuro-Dificuldades até o período de análise dos dados ou desistência ao longo do processo de aplicação da pesquisa.

\section{Instrumentos}

Teste de Criatividade Figural Infantil (TCFI; Nakano et al., 2011). Atualmente é o único teste de criatividade validado e normatizado para a faixa etária investigada. Composto por três atividades, nas quais são fornecidos estímulos incompletos a serem respondidos sob a forma de desenhos. São avaliadas 12 características criativas a partir dos desenhos realizados: fluência (número de ideias relevantes oferecidas pelo sujeito), flexibilidade (diversidade de tipos ou categorias de ideias), elaboração (adição de detalhes ao desenho básico), originalidade (ideias incomuns), expressão de emoção (expressão de sentimentos nos desenhos ou nos títulos), fantasia (presença de seres imaginários, de contos de fada ou ficção científica), movimento (expressão de movimento nos desenhos ou títulos), perspectiva incomum (desenhos realizados sob ângulos não usuais), perspectiva interna (visão interna de objetos sob a forma de transparência), uso de contexto (criação de um ambiente para o desenho), extensão de limites (estender os estímulos antes de concluir os desenhos) e títulos expressivos (ir além da descrição óbvia do desenho, abstraindo-o).

Essas características são agrupadas em quatro fatores:

1. Enriquecimento de Ideias (composto pela avaliação da elaboração nas atividades 1,2 e 3; uso de contexto nas atividades 1,2 e 3 ; perspectiva interna 
Alves, R. J. R. \& Nakano, T. C. (2015). Desempenho Criativo e suas Relações com Diferentes Medidas de Inteligência em Crianças com Dislexia do Desenvolvimento: Um Estudo Exploratório.

nas atividades 2 e 3 ; perspectiva incomum nas atividades 1,2 e 3 ; e movimento nas atividades 2 e 3),

2. Emotividade (composto pela avaliação da expressão de emoção nas atividades 1,2 e 3 ; títulos expressivos nas atividades 1,2 e 3 ; fantasia na atividades 2 e 3 ),

3. Preparação Criativa (avaliação das características elaboração, uso de contexto, movimento, títulos expressivos e perspectiva interna, todas na atividade 1),

4. Aspectos Cognitivos (composto pela avaliação da fluência nas atividades 2 e 3 ; flexibilidade nas atividades 2 e 3; originalidade nas atividades 1, 2 e 3; extensão de limites na atividade 3).

Esses fatores permitem identificar as áreas mais fortes e mais fracas do potencial criativo do indivíduo. $\mathrm{O}$ teste também oferece a obtenção de um fator geral criativo (baseado no desempenho total do teste).

Investigações de evidências de validade e precisão indicaram valores entre 0,81 e 0,94 de correlação para validade concorrente com o "Teste de Torrance - Figural", destacando-se o fato de que em todas as habilidades avaliadas foram encontrados níveis de significância de $p \leq 0,001$ e índices de precisão por meio do teste e reteste entre 0,84 e 0,95 ( $p \leq 0,001$; Nakano \& Wechsler, 2006a). Foi realizada a Análise da Variância (ANOVA) a fim de verificar influências das variáveis sexo, série, região do país, tipo de escola e da interação dessas variáveis no desempenho dos participantes, considerando-se todas as 12 características avaliadas pelo TCFI. Os resultados indicaram que as variáveis analisadas exerceram influência altamente significativa: série $(F=6,93, p \leq 0,001)$, região $(F=7,09, p \leq 0,001)$ e tipo de escola $(F=11,26, p \leq 0,001$; Nakano \& Wechsler, 2006b). Diante desses resultados, as tabelas normativas são organizadas por série escolar e tipo de escola.

Escala de Inteligência Wechsler para Crianças (WISC-III; Figueiredo, 2002). Avalia capacidade intelectual de indivíduos de seis a 16 anos e 11 meses. Compreende doze subtestes organizados em dois grupos: (a) Verbais: Informação, Semelhanças, Aritmética, Vocabulário, Compreensão, Dígitos; (b) Execução: Completar Figuras, Código, Arranjo de Figuras, Cubos, Armar Objetos, Procurar Símbolos. O desempenho no teste resulta em três medidas compostas: QI Verbal (soma dos pontos dos subtestes verbais), QI de Execução (soma dos pontos dos subtestes de Execução) e QI Total (soma dos pontos obtidos nos subtestes Verbais e de Execução). A partir do desempenho em todos os subtestes pode-se também obter quatro índices fatorais:

1. Compreensão Verbal (composto pelos subtestes Informação, Semelhanças, Vocabulário e Compreensão),

2. Organização Perceptual (composto pelos subtestes Completar Figuras, Arranjo de Figuras, Cubos e Armar Objetos),
3. Resistência à Distração (composto pelos subtestes Aritmética e Dígitos) e

4. Velocidade de Processamento (composto pelos subtestes Código e Procurar Símbolos).

Essas escalas fatoriais, assim como as escalas em QI, têm valor médio de 100 e um desvio-padrão de 15 pontos. $\mathrm{O}$ teste oferece as seguintes interpretações de desempenho intelectual: "intelectualmente deficiente", "limítrofe", "média inferior", "média", "média superior", "superior" e "muito superior". Teste originalmente elaborado por David Wechsler, em 1939, tendo-se utilizado na presente pesquisa a versão brasileira do instrumento, aprovada para uso pelo Conselho Federal de Psicologia.

Dentre os inúmeros estudos psicométricos realizados em amostras nacionais, alguns podem ser citados: estabilidade temporal por meio do procedimento teste e reteste com os subtestes "Código" e "Procurar Símbolos", verificando-se correlações consideradas fidedignas, de 0,70 e 0,63 , respectivamente; em relação à normatização, verificaram-se diferenças entre a média de três faixas etárias, por meio do procedimento da ANOVA, com valores altamente significativos ( $p<0,05)$, o que justificou a elaboração de normas de correção para o critério de idade; para validade verificaram-se validades convergentes com o teste MPCR, com valor de 0,77 , e com notas escolares, com valor moderado de correlação $(0,47)$.

Matrizes Progressivas Coloridas de Raven (MPCR; Angelini et al., 1999). Avalia o desenvolvimento intelectual (inteligência não verbal - fluida). A faixa etária de aplicação é de cinco a 11 anos e oito meses. Composto por três séries $(\mathrm{A}, \mathrm{Ab}$ e $\mathrm{B})$ ordenadas por dificuldade crescente, com 12 problemas em cada uma (figuras em que uma parte encontra-se faltando e deve ser encontrada dentre algumas alternativas), os quais somados, fornecem o escore geral. Oferece as seguintes interpretações de desempenho intelectual: "intelectualmente deficiente", "definidamente abaixo da média na capacidade intelectual", "intelectualmente médio", "definidamente acima da média na capacidade intelectual", "intelectualmente superior". Teste atualmente aprovado pelo Conselho Federal de Psicologia, desenvolvido originalmente por John C. Raven, em 1938.

Na obtenção de evidências de validade e precisão foi encontrada, por meio da ANOVA, a influência das variáveis idade, sexo, escolaridade e tipo de escola $(p \leq 0,001)$. Pela aplicação do teste " $t$ " foram ainda encontradas diferenças entre escolas públicas e particulares. Para obtenção da precisão foi utilizado o método das metades, calculando-se os coeficientes de correlação entre os itens pares e ímpares para cada sexo, em cada faixa etária, e para a amostra total. O instrumento mostrou-se preciso, com a obtenção do coeficiente corrigido, pela fórmula de Spearman-Brown, de 0,92 para o sexo masculino, de 0,90 para o sexo feminino e de 0,92 para a amostra geral. Em relação à validade foi verificada diferença no desempenho esperado entre faixas etárias, com aumentos progressivos nos resultados de acordo com o aumento da idade, como é esperado em testes de inteligência. $\mathrm{Na}$ análise da con- 
sistência interna foram verificadas correlações item-total variando entre 0,3 e 0,8 na maioria dos itens, o que indica satisfatória consistência interna do teste.

Desenho da Figura Humana (DFH-III; Wechsler, 2003). Teste validado nacionalmente e reconhecido pelo Conselho Federal de Psicologia. Avalia o desenvolvimento cognitivo em crianças de cinco a 12 anos. Por meio do desenho de uma figura masculina e outra feminina, avaliam-se 58 indicadores de desenvolvimento para cada uma delas (presença ou ausência de detalhes do corpo humano). O teste oferece as seguintes interpretações de desempenho: "deficiente", "fronteiriço", "abaixo da média", "média", "acima da média", "superior" e "muito superior". Apresenta modelo de avaliação de desenvolvimento baseado nos sistemas de Harris, Koppitz e Naglieri (Wechsler, 2003).

Em estudos nacionais de validade e precisão verificou-se, por meio da ANOVA, efeitos altamente significativos (variando entre $p \leq 0,01$ e $p \leq 0,001$ ) para as variáveis sexo, sexo da figura e faixa etária, e suas interações, o que indicou a necessidade de normas de correção separadas em relação a cada uma delas. Por meio do método do teste e reteste verificaram-se, em sua grande maioria, altas correlações (tanto no total do teste quanto por faixa etária e sexo em ambos os desenhos), que variaram de 0,22 a 0,85 $(p \leq 0,05)$, sendo indicativas de estabilidade do instrumento. $\mathrm{O}$ alfa de Cronbach indicou altos níveis de correlação entre os itens, variando de $r=0,76$ a 0,88 , demonstrando, dessa forma, ser um teste preciso. Verificou-se também a precisão do manual, no qual foi avaliado por três juízes "às cegas", encontrando-se altas correlações entre suas respostas, que variaram de 0,93 a 0,97 para o desenho da figura feminina e de 0,95 a 0,98 para o desenho da figura masculina.

\section{Procedimentos}

Primeiramente foi solicitada autorização da realização da pesquisa no Hospital das Clínicas da UNICAMP. Em seguida, o projeto foi aprovado pelo Comitê de Ética em Pesquisa da universidade ao qual o projeto encontra-se vinculado (CAAE: 13375413.8.0000.5481).

Comumente as crianças encaminhadas ao ambulatório de "Neuro-Dificuldades de Aprendizagem" apresentam como queixa principal dificuldades de aprendizagem. A princípio passam por avaliação psicológica/neuropsicológica na qual são investigadas Funções Corticais Superiores, tais como inteligência, funções executivas, habilidades gerais de linguagem, desempenho escolar, desempenho psicomotor, entre outras. Os testes de inteligência utilizados no estudo aqui relatado (MPCR, WISC-III e DFH-III) já faziam parte do protocolo de aplicação dos psicólogos da equipe, tendo sido adicionado somente o de criatividade (TCFI). Especificamente para aplicação desses quatro testes foram utilizadas quatro sessões individuais de 50 minutos com cada criança.

Após essa primeira etapa de atendimento do ambulatório, o procedimento comum adotado envolve, naqueles casos em que são encontrados sinais psicológicos/neuro- psicológicos característicos da dislexia, encaminhamento para avaliação de outros profissionais, dentre eles fonoaudiólogos, psicopedagogos, neurologistas, psiquiatras, fisioterapeutas, sendo que, após essas avaliações, o diagnóstico é finalizado, em conjunto pela equipe, de modo interdisciplinar. Salienta-se que, para todas as avaliações feitas pelo ambulatório, a autorização prévia dos pais/ responsáveis faz parte do processo de diagnóstico.

Ao longo do processo de coleta de dados, naqueles casos cujo diagnóstico de dislexia foi confirmado, os pais/responsáveis foram contatados, sendo marcada uma reunião. Nessa, o objetivo da pesquisa foi apresentado, sendo solicitada autorização para utilização dos dados já coletados durante a avaliação psicológica/neuropsicológica. Em caso positivo, foi solicitada a assinatura do "Termo de Consentimento Livre e Esclarecido".

Para a análise dos dados foi utilizado o programa SPSS Statistics 20.0 for Windows ${ }^{\circledR}$ (Statistical Package for Social Sciences; SPSS Inc., Chicago, IL, USA, 2008). Por meio da estatística descritiva foram obtidos dados de frequência, média, desvio padrão, pontuação mínima e máxima. Dado o tamanho da amostra e a ausência de distribuição normal, os tipos de análise de estatística inferencial selecionadas foram todas do tipo não paramétrica.

\section{Resultados}

A primeira análise realizada referiu-se ao desempenho do grupo no teste de criatividade. Por meio da Prova $F$ de Friedman verificou-se que não houve diferenças significativas entre as médias obtidas nos quatro fatores $\left(\chi^{2}=3,46\right.$; $p=0,32)$ do TCFI, considerando-se o valor do percentil. A estatística descritiva para cada um desses fatores e do total do instrumento pode ser visualizada na Tabela 1.

No Fator 1 verifica-se que a maior parte da amostra (38\%) apresentou classificação dentro da média, o mesmo ocorre com o Fator 4 - Aspectos Cognitivos (54\%). No Fator 3 (Preparação Criativa) observa-se que $46 \%$ da amostra apresentou classificação "inferior", e, inverso a isso, no Fator 2 (Emotividade) observa-se que a mesma porcentagem da amostra apresentou a classificação "superior". No total do teste a classificação "média" foi a mais frequente (31\%), sendo que o restante da amostra praticamente divide-se entre as outras classificações, de modo que, na presente amostra, de um modo geral, as crianças com dislexia não apresentaram destaque criativo quando comparadas com aquelas que compuseram a amostra normativa do teste. A exceção ocorre em quatro participantes (30,7\%), os quais apresentaram classificação superior ou acima da média.

Na Tabela 2 pode ser observada a estatística descritiva obtida nos testes de inteligência. Analisando-se separadamente os resultados de cada instrumento, pode-se verificar que no teste MPCR a maioria da amostra apresentou desempenho dentro da média (54\%), e o restante dividiu-se entre as classificações "superior" e "abaixo da média" (ambas com 23\%). Desempenho superior pode ser notado em 3 participantes. 
Alves, R. J. R. \& Nakano, T. C. (2015). Desempenho Criativo e suas Relações com Diferentes Medidas de Inteligência em Crianças com Dislexia do Desenvolvimento: Um Estudo Exploratório.

Tabela 1

Estatística Descritiva do Desempenho no Teste TCFI

\begin{tabular}{|c|c|c|c|c|c|c|c|}
\hline Itens do TCFI & & & & & & Classificação & $f(\%)$ \\
\hline \multirow{5}{*}{$\begin{array}{l}\text { Fator } 1 \\
\text { Enriquecimento de } \\
\text { Ideias }\end{array}$} & $M$ & $D P$ & $M d n$ & Min & Max & Superior & $2(15)$ \\
\hline & \multicolumn{5}{|c|}{ Pontos Brutos } & Acima da Média & $2(15)$ \\
\hline & 40,15 & 19,60 & 42,00 & 11 & 75 & Média & $5(38)$ \\
\hline & \multicolumn{5}{|c|}{ Percentil } & Abaixo da Média & $0(0)$ \\
\hline & 45,69 & 31,43 & 46,00 & 2 & 92 & Inferior & $4(31)$ \\
\hline \multirow{5}{*}{$\begin{array}{l}\text { Fator } 2 \\
\text { Emotividade }\end{array}$} & $M$ & $D P$ & $M d n$ & Min & Max & Superior & $6(46)$ \\
\hline & \multicolumn{5}{|c|}{ Pontos Brutos } & Acima da Média & $2(15)$ \\
\hline & 6,92 & 8,04 & 5,00 & 0 & 25 & Média & $0(0)$ \\
\hline & \multicolumn{5}{|c|}{ Percentil } & Abaixo da Média & $1(8)$ \\
\hline & 60,54 & 35,93 & 79,00 & 10 & 97 & Inferior & $4(31)$ \\
\hline \multirow{5}{*}{$\begin{array}{l}\text { Fator } 3 \\
\text { Preparação Criativa }\end{array}$} & $M$ & $D P$ & $M d n$ & Min & Max & Superior & $1(8)$ \\
\hline & \multicolumn{5}{|c|}{ Pontos Brutos } & Acima da Média & $1(8)$ \\
\hline & 4,31 & 4,11 & 3,00 & 0 & 13 & Média & $3(23)$ \\
\hline & \multicolumn{5}{|c|}{ Percentil } & Abaixo da Média & $2(15)$ \\
\hline & 30,46 & 28,73 & 23,00 & 2 & 86 & Inferior & $6(46)$ \\
\hline \multirow{5}{*}{$\begin{array}{l}\text { Fator } 4 \\
\text { Aspectos Cognitivos }\end{array}$} & $M$ & $D P$ & $M d n$ & Min & Max & Superior & $1(8)$ \\
\hline & \multicolumn{5}{|c|}{ Pontos Brutos } & Acima da Média & $0(0)$ \\
\hline & 46,15 & 12,36 & 49,00 & 22 & 68 & Média & $7(54)$ \\
\hline & \multicolumn{5}{|c|}{ Percentil } & Abaixo da Média & $3(23)$ \\
\hline & 42,23 & 23,97 & 39,00 & 6 & 89 & Inferior & $2(15)$ \\
\hline \multirow{5}{*}{ Pontuação Total } & $M$ & $D P$ & $M d n$ & Min & Max & Superior & $2(15)$ \\
\hline & \multicolumn{5}{|c|}{ Pontos Brutos } & Acima da Média & $2(15)$ \\
\hline & 99,08 & 35,17 & 99,00 & 34 & 155 & Média & $4(31)$ \\
\hline & \multicolumn{5}{|c|}{ Percentil } & Abaixo da Média & $3(23)$ \\
\hline & 48,38 & 30,18 & 48 & 2 & 95 & Inferior & $2(15)$ \\
\hline
\end{tabular}

Nota. Legenda: M: Média; DP: Desvio Padrão; Mdn: Mediana; Min: Mínimo; Max: Máximo; f: Frequência.

Também no teste DFH-III a maioria da amostra apresentou classificação dentro da média (54\%) no resultado total, com duas crianças ( $15 \%$ da amostra) apresentando desempenho "fronteiriço". Na figura feminina, a maioria do grupo (62\%) obteve a classificação "média", sendo que, em relação à figura masculina, há divisão entre classificação "média" e "abaixo da média", ambas concentrando $39 \%$ dos estudantes. O Teste de Wilcoxon não indicou diferença entre as médias dos pontos brutos da figura feminina e masculina $(Z=-0,47 ; p=0,63)$.

A análise do desempenho na WISC-III, por sua vez, é apresentada de acordo com as escalas avaliadas no instrumento (verbal, execução, compreensão verbal, organização perceptual, resistência à distração e velocidade de processamento) e sua pontuação total. Em todas essas escalas observa-se que a classificação "média" foi obtida pela maioria dos participantes. Especificamente no total do teste, observa-se que $54 \%$ obteve a classificação "média", fazendo-se notar ainda $23 \%$ na classificação "médio superior" e $15 \%$ com resultado "superior". Somente um participante apresentou desempenho "médio inferior". Novamente pode-se notar a presença de estudantes com destaque em seu desempenho $(n=5)$, nesse caso, cognitivo, aliado ao quadro da dislexia. 
Psicologia: Reflexão e Crítica, 28(2), 280-291.

Tabela 2

Estatística Descritiva do Desempenho nos Testes MPCR, DFH-III e WISC-III

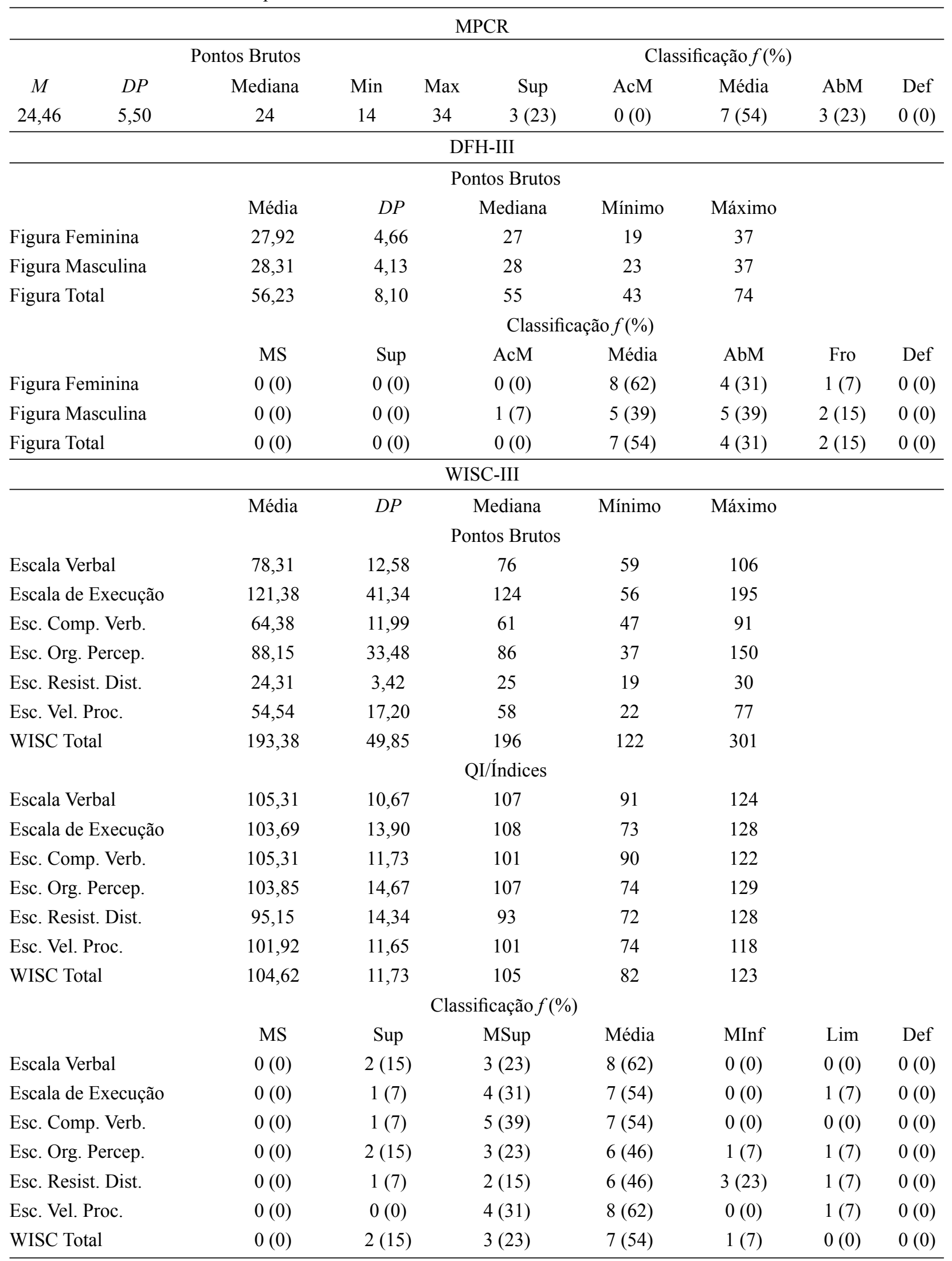

Nota. Legenda: DP: Desvio Padrão; $f$ : Frequência; MS: Muito Superior; Sup: Superior; AcM: Acima da Média; AbM: Abaixo da Média; Fro: Fronteiriço; Lim: Limítrofe; Def: Deficiente; Esc. Comp. Verb.: Escala de Compreensão Verbal; Esc. Org. Percep.: Escala de Organização Perceptual; Esc. Resist. Dist.: Escala de Resistência à Distração; Esc. Vel. Proc.: Escala de Velocidade de Processamento; MSup: Médio Superior; MInf: Médio Inferior. 
Alves, R. J. R. \& Nakano, T. C. (2015). Desempenho Criativo e suas Relações com Diferentes Medidas de Inteligência em Crianças com Dislexia do Desenvolvimento: Um Estudo Exploratório.

O Teste de Wilcoxon indicou que não houve diferenças significativas entre as médias do QI Verbal e do QI de Execução $(Z=-0,69 ; p=0,48)$. Por meio da Prova $F$ de Friedman também foi possível verificar que não houve diferenças significativas entre as médias dos quatro Índices Fatoriais (compreensão verbal, organização perceptual, resistência à distração e velocidade de processamento; $\chi^{2}=3,83 ; p=0,28$ ).

A análise a seguir teve como objetivo estimar a correlação entre as medidas de criatividade e de inteligência, cujos resultados encontram-se disponíveis na Tabela 3. Os dados mostraram que, em sua maioria, não foram verificadas correlações significativas entre as medidas, como, por exemplo, entre o teste DFH-III (desenho da figura masculina, feminina e total) e as escalas da WISC-III (verbal, execução, compreensão verbal, organização perceptual, resistência à distração, velocidade de processamento e seu total) com os quatro fatores e total do TCFI. O mesmo ocorre entre o MPCR e os fatores 3 e 4 do TCFI. As exceções se fazem em relação ao MPCR e o Fator $1(r=0,810$, $p=0,001)$, Fator $2(r=0,616, p=0,025)$ e a pontuação total do TCFI $(r=0,801, p=0,001)$, que apresentaram correlações significativas e positivas.

Tabela 3

Matriz de Correlação entre as Pontuações Brutas dos Testes TCFI, DFH-III, WISC-III e MPCR

\begin{tabular}{|c|c|c|c|c|c|c|}
\hline & & F1 & F2 & F3 & F4 & $\mathrm{TC}$ \\
\hline \multirow{2}{*}{$\mathrm{FgF}$} & $r$ & 0,114 & 0,199 & 0,215 & $-0,111$ & 0,203 \\
\hline & $p$ & 0,712 & 0,514 & 0,481 & 0,719 & 0,505 \\
\hline \multirow{2}{*}{$\mathrm{FgM}$} & $r$ & 0,377 & 0,331 & 0,520 & $-0,330$ & 0,441 \\
\hline & $p$ & 0,204 & 0,269 & 0,068 & 0,271 & 0,131 \\
\hline \multirow{2}{*}{ DFH } & $r$ & 0,262 & 0,260 & 0,398 & $-0,217$ & 0,334 \\
\hline & $p$ & 0,387 & 0,392 & 0,178 & 0,477 & 0,265 \\
\hline \multirow{2}{*}{ Ver } & $r$ & 0,052 & 0,331 & 0,289 & 0,028 & 0,083 \\
\hline & $p$ & 0,865 & 0,269 & 0,339 & 0,929 & 0,789 \\
\hline \multirow{2}{*}{ Exe } & $r$ & 0,281 & 0,091 & 0,367 & 0,290 & 0,212 \\
\hline & $p$ & 0,352 & 0,768 & 0,217 & 0,337 & 0,486 \\
\hline \multirow{2}{*}{ CVW } & $r$ & 0,083 & 0,361 & 0,268 & 0,102 & 0,138 \\
\hline & $p$ & 0,788 & 0,225 & 0,376 & 0,739 & 0,652 \\
\hline \multirow{2}{*}{ OPW } & $r$ & 0,353 & 0,292 & 0,396 & 0,344 & 0,339 \\
\hline & $p$ & 0,237 & 0,333 & 0,181 & 0,249 & 0,257 \\
\hline \multirow{2}{*}{ RDW } & $r$ & 0,262 & $-0,037$ & 0,076 & 0,105 & 0,179 \\
\hline & $p$ & 0,388 & 0,903 & 0,805 & 0,733 & 0,558 \\
\hline \multirow{2}{*}{ VPW } & $r$ & 0,039 & $-0,065$ & 0,239 & $-0,190$ & $-0,041$ \\
\hline & $p$ & 0,901 & 0,833 & 0,432 & 0,535 & 0,894 \\
\hline \multirow{2}{*}{ Wis } & $r$ & 0,017 & 0,079 & 0,178 & 0,050 & $-0,019$ \\
\hline & $p$ & 0,957 & 0,797 & 0,562 & 0,872 & 0,950 \\
\hline \multirow{2}{*}{ Rav } & $r$ & $0,810^{* *}$ & $0,616^{*}$ & 0,117 & 0,076 & $0,801^{* *}$ \\
\hline & $p$ & 0,001 & 0,025 & 0,704 & 0,805 & 0,001 \\
\hline
\end{tabular}

Nota. Legenda: $r$ : Coeficiente de correlação de Sperman; $p$ : Valor de $p$; * Valor significativo; ** Valor altamente significativo; F1: Fator 1 do TCFI; F2: Fator 2 do TCFI; F3: Fator 3 do TCFI; F4: Fator 4 do TCFI; TC: Total do TCFI; FgF: Figura Feminina do DFH-III; FgM: Figura Masculina do DFH-III; DFH: Pontuação Total do DFH-III; Ver: Escala Verbal WISC-III; Exe: Escala de Execução WISC-III; CVW: Escala de Compreensão Verbal WISC-III; OPW: Escala de Organização Perceptual WISC-III; RDW: Escala de Resistência à Distração WISC-III; Wis: Total do WISC-III; Rav: Total do MPCR. 


\section{Discussão}

De acordo com os resultados encontrados foi possível verificar, de modo geral, que as crianças com Dislexia do Desenvolvimento envolvidas no presente estudo, apresentaram um desempenho em criatividade dentro da média. Do mesmo modo também não foi verificado baixo desempenho, visto que a amostra praticamente dividiu-se entre as classificações, de forma a confirmar alguns resultados encontrados na literatura científica, tais como os relatados por Tafti et al. (2009), em que não foi verificado desempenho estatisticamente diferente entre crianças com dislexia daquelas sem dificuldades escolares quando utilizado o teste figural de Torrance.

Lockiewicz, Bogdanowicz e Bogdanowicz (2013) ao investigarem habilidades visoespaciais, motivação e criatividade em adultos com dislexia e adultos que apresentavam uma vida acadêmica satisfatória, relataram que os resultados não apontaram diferenças significativas entre os grupos no desempenho de tarefas de habilidades visoespaciais e criatividade, diferentemente do que ocorreu com a motivação, mais evidenciada no grupo com dislexia. Os autores especularam que as produções criativas cotidianas muitas vezes atribuídas aos que apresentam dislexia poderiam ter maior ligação com aspectos emocionais, inclusa a motivação, e até mesmo com características de personalidade, e não com fatores cognitivos. Tal percepção vai ao encontro do resultado obtido na presente pesquisa, visto que o melhor desempenho dos participantes se dá no Fator 2 (Emotividade), no qual um número importante de classificações na faixa "superior" foi encontrado.

Nesse sentido, destaque deve ser dada à posição de Lubart (2007) ao afirmar que os aspectos emocionais são fundamentais para produção criativa. Para ele, três hipóteses a esse respeito podem ser feitas: (a) a expressão das emoções relativa às experiências pessoais poderia ser o motor de uma produção criativa, (b) a emoção poderia colocar o indivíduo em estado mental motivacional propício à criatividade, $(\mathrm{c})$ os aspectos emocionais poderiam servir para ajudar os indivíduos a selecionarem as ideias mais promissoras. Dessa maneira, "as emoções desempenhariam um papel central dentro do processo de formação de associações criativas" (p. 56), fazendo-se notar, em relação à temática da criatividade, uma tendência em reconhecer e valorizar a influência dos aspectos emocionais na expressão criativa (Nakano \& Zaia, 2012).

Importante destacar que, historicamente, autores reconhecidos no estudo da criatividade (como Torrance, Sternberg, Amabile, Csikszentmihalyi e Gardner) já apontavam para a importância dos aspectos emocionais da criatividade enquanto elementos complementares aos aspectos cognitivos (fluência, flexibilidade, elaboração e originalidade), tidos inicialmente como únicos representantes e componentes do processo criativo (Sternberg \& Grigorenko, 2001). Sobre essa questão, Zenasni e Lubart (2008) realizaram uma revisão sistemática de estudos que examinaram a relação entre emoção e criatividade, e verificaram que a maioria deles focou o impacto direto do estado emocional na criatividade, apontando que um estado positivo favoreceria a criatividade e estados negativos funcionariam como inibidor. Nesse sentido destaque deve ser dado ao fato de que o teste utilizado somente pontua a presença de emoções nas respostas dos sujeitos, sem determinar sua natureza (positiva ou negativa), de modo que um estudo futuro com esse propósito mostra-se bastante interessante.

Salienta-se, entretanto, na literatura, a frequente constatação de que nos casos de dislexia faz-se presente uma maior carga de sensibilidade emocional negativa (comumente, maior frequência de depressão e transtornos de ansiedade; Alexander-Passe, 2006; Nelson \& Gregg, 2012), de modo que tal resultado abre margem para maiores discussões acerca de como as emoções, independentemente de sua natureza, poderiam influenciar o desempenho criativo.

Especificamente em relação à investigação da dupla excepcionalidade (dislexia e criatividade), foi possível verificar que, na amostra considerada, quatro participantes $(30,7 \%)$ apresentaram classificação superior ou acima da média no teste que avalia a criatividade, de modo a poder serem classificados dentro desse padrão cognitivo. Dada a escassez de estudos voltados à essa temática e ao reconhecimento tardio da existência desse padrão cognitivo científica e clinicamente (Schultz, 2009), a pesquisa aqui apresentada adquire importante valor, ainda que pesquisas mais amplas sejam recomendadas considerando-se que a identificação desse grupo é complicada devido ao fato de que suas habilidades bem desenvolvidas podem atuar de modo a mascarar seus processos psicológicos deficitários e seus problemas de aprendizagem, conforme exposto por Pfeiffer (2013).

Para os testes de inteligência aplicados foi verificada a classificação "média" na maioria deles. Tais resultados eram esperados, visto que um dos critérios diagnósticos para dislexia é apresentar inteligência dentro ou acima da média (APA, 2002). Especificamente no DFH-III dois sujeitos apresentaram a classificação "fronteiriço", que iria contra esse critério, devendo-se destacar que tal situação ocorreu exclusivamente neste instrumento, não se repetindo em relação ao total dos demais instrumentos de avaliação da inteligência utilizados. Não foi vista também nenhuma classificação "deficiente" em nenhum dos totais dos três testes.

Historicamente, o teste DFH possui algumas vertentes sobre qual seria a maneira mais adequada de interpretá-lo, destacando-se, dentre elas, como medida de desenvolvimento infantil (adotado no presente trabalho), medida projetiva e como passível de avaliação de aspectos emocionais (Hutz \& Bandeira, 1995). Convém salientar, no entanto, que não foram encontrados estudos especificamente utilizando um grupo com dislexia enquanto critério para o primeiro modelo de interpretação. Alguns estudos indicaram que, em amostras regulares, a ausência de indicadores considerados desenvolvimentais poderia ser indicadora de problemas emocionais e de dificuldades 
de aprendizagem (Arteche, 2006; Bartholomeu, Sisto, \& Rueda, 2006). Desse modo, esses achados na literatura poderiam explicar os resultados aqui encontrados para a presença da classificação "fronteiriço" encontrado em duas crianças com dislexia, tomando os aspectos emocionais e as dificuldades de aprendizagem envolvidas nesse quadro, situação que merece ser melhor investigada em um estudo futuro envolvendo a avaliação dos aspectos emocionais, ainda não validada no sistema utilizado no instrumento.

$\mathrm{Na}$ investigação da relação entre criatividade e inteligência, podem ser citadas primeiramente as fortes correlações encontradas entre o total do teste MPCR e o TCFI. Ao pressupor o teste MPCR enquanto uma medida relacionada à inteligência fluida, resultados semelhantes são encontrados na literatura (Batey, Chamorro-Premuzic, \& Furnham, 2009; Preckel et al., 2006; Sligh, Conners, \& Roskos-Ewoldsen, 2005), fazendo uso de uma população geral, sem o quadro da dislexia. Do mesmo modo também são encontrados resultados contrários (Furnham, Batey, Anand, \& Manfield, 2008; Reuter et al., 2005), de modo a revelar uma inconstância nos achados sobre esses construtos. Não foram encontrados estudos que abordassem especificamente a investigação de ambos os construtos em grupos de indivíduos com dislexia.

Por outro lado, tanto o DFH-III quanto as subescalas da WISC-III não apresentaram correlação significativa com nenhuma medida do instrumento de criatividade. Especialmente em relação ao DFH-III e o TCFI, os resultados divergem dos que foram relatados por Nakano (2012), que, ao fazer uso dos mesmos instrumentos, mas em uma amostra de estudantes regulares, relatou correlação moderada de 0,47 entre as medidas. Em relação à WISC, Parisi et al. (2007) também verificaram correlações positivas com o teste de Torrance em crianças nascidas com baixo peso.

Estudos envolvendo o instrumento de criatividade utilizado na pesquisa atual apontam claramente para a influência de diversos fatores (amostra e instrumento utilizado) em seus resultados. Pesquisas fazendo uso do mesmo instrumental, também com a finalidade de correlacionar criatividade e inteligência, indicaram resultados bastante diferentes, dependendo do instrumento de inteligência utilizado. Como pode ser visto, quando esse construto foi avaliado a partir de um instrumento de desenvolvimento cognitivo, Desenho da Figura Humana (Nakano, 2012), a correlação entre os desempenhos mostrou-se moderada $(r=0,47)$, devendo-se atentar ao fato de que tal valor pode ter sido influenciado pela similaridade da tarefa a ser realizada nos dois instrumentos (desenhos). Correlação baixa $(r=0,22, p<0,008)$ entre o desempenho no TCFI e na Bateria de Provas de Raciocínio Infantil foi relatada por Nakano e Brito (2013). No entanto, outros estudos relacionando o teste de criatividade com outras medidas de inteligência apresentaram ausência de correlação significativa, como quando correlacionando o desempenho no TCFI com a versão padrão da Bateria Woodcock-Johnsson III (Chiodi, Nakano, \& Wechsler, 2011a), quanto com sua versão ampliada (Chiodi, Nakano, \& Wechsler, 2011b).
Tal constatação vem reforçar algumas considerações que vêm sendo apontadas na literatura nacional e internacional (K. H. Kim, 2005; Preckel et al., 2006; Wechsler et al., 2010) acerca das relações entre criatividade e inteligência: após um grande número de pesquisas desenvolvidas, o consenso está longe de ser atingido. Dessa forma, apesar de ser um tema bastante estudado, as investigações atuais seguem questionando a existência dessa relação e o nível da associação, além da sua estabilidade no tempo e possibilidade de generalização para populações distintas (Elisondo \& Donolo, 2010). Tal padrão inconstante repetiu-se nos resultados encontrados aqui para a população com Dislexia do Desenvolvimento.

\section{Considerações Finais}

Os resultados encontrados nessa pesquisa indicaram um desempenho, de forma geral, dentro da média para o grupo com dislexia, tanto nas medidas de criatividade quanto nas de inteligência, assim como não foi encontrado um padrão nas correlações entre criatividade e inteligência nesse grupo. Embora a maior parte daqueles estudos encontrados na literatura tenha concluído acerca da existência de alguma relação entre criatividade e inteligência, com a amplitude desta relação variando entre baixa e moderada, as diferenças relatadas acabam dificultando o consenso em relação a esta questão. Os achados, aparentemente contraditórios, podem ser explicados, de acordo com Mouchiroud e Lubart (2002), pelo tipo de estudo, pela heterogeneidade das medidas empregadas e o tipo de população estudada. Neste sentido, a importância de se refletir sobre o tema e procurar respostas que determinem a existência ou não desta relação pode enriquecer, por exemplo, as discussões dentro da temática das altas habilidades, uma vez que, dentro dos modelos mais atuais, esta teria, dentre seus componentes, a inteligência e criatividade (Renzulli, 2008).

Os resultados ainda apontaram quatro participantes que apresentaram classificação superior ou acima da média em criatividade, de modo a se poder hipotetizar acerca da presença da dupla excepcionalidade em $30,7 \%$ da amostra. A importância dessa identificação ampara-se, segundo Kalbfleisch e Iguchi (2008), na constatação de que cerca de $41 \%$ dos estudantes que apresentam os quadros combinados usualmente não são diagnosticados até a faculdade. Nesse sentido, pesquisas voltadas à prevalência desses estudantes no sistema educacional brasileiro, assim como aquelas voltadas a procedimentos de identificação, intervenções e políticas educacionais devem ser incentivadas.

Como sugestão para futuras investigações propõe-se que sejam realizados estudos voltados à avaliação de outros construtos psicológicos/neuropsicológicos que possam permear asrelações entre criatividade e inteligência na Dislexia do Desenvolvimento. Por exemplo, investigar as relações entre aspectos emocionais, de personalidade, funções executivas, com ambos os construtos abordados na presente pesquisa, de modo a verificar se suas correlações possam oferecer possibilidades de explicação para os resultados aqui encontrados. 
Convém destacar também que a presente pesquisa representou um importante avanço para a temática, visto que foi feito uso de instrumentos cientificamente reconhecidos para avaliação da criatividade, fato que raramente ocorreu nos estudos internacionais que investigaram criatividade na dislexia (Alves \& Nakano, no prelo). Em relação ao tamanho da amostra, convém salientar a dificuldade em se conseguir participantes que atendessem aos rígidos critérios utilizados pelo local onde os dados foram coletados, fato que, se por um lado, inviabilizou a obtenção de um maior número de sujeitos, por outro, ofereceu aos pesquisadores maior segurança nos resultados obtidos. No entanto, não se pode deixar de considerar que, usualmente, estudos que trabalham com populações clínicas, apresentam limitações amostrais, haja vista a dificuldade de avaliação diagnóstica em tais populações. Tal fato acaba por comprometer o potencial de generalização dos resultados encontrados na presente pesquisa, de modo que bastante cautela é indicada na interpretação dos mesmos, limitando-se, por ora, aos indivíduos participantes. Sugere-se, desse modo, a realização de futuras investigações com amostragem maior, a fim de que se possa obter resultados mais generalizáveis para a população em questão.

Dada à escassez de estudos voltados a essa temática, a pesquisa aqui apresentada adquire importante valor científico, ainda que pesquisas mais amplas sejam recomendadas. Em médio e longo prazo espera-se que o estudo da temática seja propulsora de possibilidades para que indivíduos com dislexia encontrem satisfação pessoal e profissional, bem como tenham seus interesses respeitados e suas habilidades valorizadas a fim de que o diagnóstico não atue de forma impeditiva ao seu desenvolvimento. Os ganhos não serão somente em nível individual, mas também social, dada a oportunidade desses sujeitos exercitarem sua criatividade de forma a contribuir para a solução dos problemas atuais.

Por conseguinte, aponta-se que a criatividade aqui investigada restringiu-se à criatividade figurativa, de modo que outros estudos envolvendo outros tipos de criatividade, como a verbal, poderão confirmar ou não os resultados aqui apresentados, dada a complexidade desse construto e sua multidimensionalidade (Wechsler, 2008).

\section{Referências}

Alexander-Passe, N. (2006). How dyslexic teenagers cope: An investigation of self-esteem, coping and depression. Dyslexia, 12(4), 256-275. doi:10.1002/dys.318

Alves, R. J. R., \& Nakano, T. C. (no prelo). Criatividade em portadores de transtornos e dificuldades de aprendizagem: Revisão de pesquisas. Psicologia Escolar e Educacional.

American Psychiatric Association. (2002). DSM-IV-TR: Manual diagnóstico e estatístico de transtornos mentais (ed. rev.). Porto Alegre, RS: Artes Médicas.

Angelini, A. L., Alves, I. C. B., Custódio, E. M., Duarte, W. F., \& Duarte, J. L. M. (1999). Matrizes Progressivas Coloridas de Raven: Escala Especial. São Paulo, SP: Centro Editor de Testes e Pesquisa em Psicologia.
Arteche, A. X. (2006). Indicadores emocionais do desenho da figura humana: Construção e validação de uma escala infantil (Tese de doutorado, Universidade Federal do Rio Grande do Sul, Porto Alegre, RS, Brasil).

Bartholomeu, D., Sisto, F. F., \& Rueda, F. J. M. (2006). Dificuldades de aprendizagem na escrita e características emocionais de crianças. Psicologia em Estudo, 11(1), 139-146. doi:10.1590/ S1413-73722006000100016

Batey, M., Chamorro-Premuzic, T., \& Furnham, A. (2009). Intelligence and personality as predictors of divergent thinking: The role of general, fluid and crystallized intelligence. Thinking Skills and Creativity, 4(1), 60-69. doi:10.1016/j. tsc.2009.01.002

Brunswick, N., Martin, G. N., \& Marzano, L. (2010). Visuospatial superiority in developmental dyslexia: Myth or reality? Learning and Individual Differences, 20(5), 421-426. doi:10.1016/j.lindif.2010.04.007

Chakravarty, A. (2009). Artistic talent in dyslexia - A hypothesis. Medical Hypotheses, 73(4), 569-571. doi:10.1016/j. mehy.2009.05.034

Chiodi, M. G., Nakano, T. C., \& Wechsler, S. M. (2011a). Estudo de correlação entre criatividade e habilidades intelectuais. Trabalho apresentado no I Congresso Internacional de Criatividade: Inovação, Manaus, AM, Brasil.

Chiodi, M. G., Nakano, T. C., \& Wechsler, S. M. (2011b). Inteligência e criatividade: Um estudo correlacional. Trabalho apresentado no I Congresso Internacional de Criatividade: Inovação, Manaus, AM, Brasil.

Cohn, R., \& Neumann, M. A. (1977). Artistic production in dyslectic children. Neurología, Neurocirugía, Psiquiatria, 18(2-3), 65-69.

Çorlu, M., Özcan, O., \& Korkmazlar, Ü. (2007). The potential of dyslexic individuals in communication design education. Behavioural Neurology, 18(4), 217-223. doi: $10.1155 / 2007 / 327530$

Çorlu, M., Özcan, O., \& Korkmazlar, Ü. (2009). The meaning of dyslexic's drawings in communication design. Dyslexia, 15(2), 148-154. doi:10.1002/dys.362

Davis, G. A., Rimm, S. B., \& Siegle, D. (2011). Education of the gifted and talented $\left(6^{\text {th }}\right.$ ed.). Upper Saddle River, NJ: Pearson.

Eide, B. L., \& Eide, F. F. (2011). The dyslexic advantage: Unlocking the hidden potential of the dyslexic brain. New York: Hay House.

Elisondo, R. C., \& Donolo, D. S. (2010). ¿Creatividad o inteligencia? That is not the question. Anales de Psicologia, 26(2), 220-225.

Everatt, J., Steffert, B., \& Smythe, I. (1999). An eye for the unusual: Creative thinking in dyslexics. Dyslexia, 5(1), 28-46. doi:10.1002/(SICI)1099-0909(199903)5:1<28::AID-DYS126>3.0.CO;2-K

Figueiredo, V. L. M. (2002). WISC-III: Escala de Inteligência Wechsler para crianças. São Paulo, SP: Casa do Psicólogo.

Furnham, A., Batey, M., Anand, K., \& Manfield, J. (2008). Personality, hypomania, intelligence and creativity. Personality and Individual Differences, 44(5), 1060-1069. doi:10.1016/j. paid.2007.10.035

Guilford, J. P. (1967). The nature of human intelligence. New York: McGraw-Hill.

Hutz, C. S., \& Bandeira, D. R. (1995). Avaliação psicológica com o desenho da figura humana: Técnica ou intuição? Temas em Psicologia, 3(3), 35-41.

Kalbfleisch, M. L., \& Iguchi, C. M. (2008). Twice-exceptional learners. In J. A. Plucker \& C. M. Callahan (Eds.), Critical 
Alves, R. J. R. \& Nakano, T. C. (2015). Desempenho Criativo e suas Relações com Diferentes Medidas de Inteligência em Crianças com Dislexia do Desenvolvimento: Um Estudo Exploratório.

issues and practices in gifted education (pp. 707-720). Waco, TX: Prufrock Press.

Károlyi, C., \& Winner, E. (2004). Dyslexia and visual spatial talents: Are they connected? Students with Both Gifts and Learning Disabilities, 95-117. doi:10.1007/978-1-4419-9116-4_6

Kim, J.-Y., \& Ko, Y.-G. (2007). If gifted/learning disabled students have wisdom, they have all things! Roeper Review, 29(4), 249-258. doi:10.1080/02783190709554419

Kim, K. H. (2005). Can only intelligent people be creative? A meta-analysis. Prufrock Journal, 16(2-3), 57-66. doi:10.4219/ jsge-2005-473

Lockiewicz, M., Bogdanowicz, K. M., \& Bogdanowicz, M. (2013). Psychological resources of adults with developmental dyslexia. Journal of Learning Disabilities, 20(10), 1-13. doi:10.1177/0022219413478663

Lubart, T. (2007). Psicologia da criatividade (M. C. M. Moraes, Trad.). Porto Alegre, RS: Artmed (Original publicado em 2003)

Lyon, G. R., Shaywitz, S. E., \& Shaywitz, B. A. (2003). A definition of dyslexia. Annals of Dyslexia, 53(1), 1-14. doi:10.1007/ s11881-003-0001-9

Mouchiroud, C., \& Lubart, T. (2002). Social creativity: A cross-sectional study of 6 to 11 year-old children. International Journal of Behavioral Development, 26(1), 60-69.

Nakano, T. C. (2012). Criatividade e inteligência em crianças: Habilidades relacionadas? Psicologia: Teoria e Pesquisa, 28(2), 149-159. doi:10.1590/S0102-37722012000200003

Nakano, T. C., \& Brito, M. E. (2013). Avaliação da criatividade a partir do controle do nível de inteligência em uma amostra de crianças. Temas em Psicologia, 21(1), 1-15. doi:10.9788/ tp2013.1-01

Nakano, T. C., \& Wechsler, S. M. (2006a). Teste brasileiro de criatividade figural: Proposta de instrumento. Interamerican Journal of Psychology, 40(1), 103-110.

Nakano, T. C., \& Wechsler, S. M. (2006b). Teste brasileiro de criatividade figural: Proposta de normas. Avaliação Psicológica, 5(2), 159-170.

Nakano, T. C., Wechsler, S. M., \& Primi, R. (2011). Teste de Criatividade Figural Infantil. São Paulo, SP: Vetor.

Nakano, T. C., \& Zaia, P. (2012). Criatividade e inteligência emocional em crianças: Um estudo relacional. Psico, 43(3), 388-399.

Nelson, J. M., \& Gregg, N. (2012). Depression and anxiety among transitioning adolescents and college students with ADHD, dyslexia, or comorbid ADHD/dyslexia. Journal of Attention Disorders, 16(3), 244-254. doi:10.1177/1087054710385783

Neumeister, K. S., Yssel, N., \& Burney, V. H. (2013). The influence of primary caregivers in fostering success in twice-exceptional children. Gifted Child Quarterly, 57(4), 263-274. doi:10.1177/0016986213500068

Noronha, A. P. P., Primi, R., \& Alchieri, J. C. (2005). Instrumentos de avaliação mais conhecidos/utilizados por psicólogos e estudantes de Psicologia. Psicologia: Reflexão e Crítica, 18(3), 390-401. doi:10.1590/S0102-79722005000300013

Organização Mundial da Saúde. (2008). Classificação de transtornos mentais e de comportamento da CID-10: Descrição clínicas e diretrizes diagnósticas (ed. rev.). Porto Alegre, RS: Artes Médicas.

Pachalska, M., Bogdanowicz, K., Tomaszewska, K., Łockiewicz, M., \& Bogdanowicz, M. (2009). The stimulation of creative activity in dyslexic adults. Acta Neuropsychologica, 7(2), 113-130.

Parisi, L., Di Filippo, T., Firrigno, L., La Grutta, S., Testa, D., \& Roccella, M. (2007). Description and evaluation of crea- tive thinking in preterm low birth weight infants. Minerva Pediatrica, 59(2), 121.

Pfeiffer, S. I. (2013). Serving the gifted: Evidence-based clinical and psychoeducational practice. New York: Routledge.

Preckel, F., Holling, H., \& Wiese, M. (2006). Relationship of intelligence and creativity in gifted and non-gifted students: An investigation of threshold theory. Personality and Individual Differences, 40(1), 159-170. doi:10.1016/j.paid.2005.06.022

Renzulli, J. S. (2008). La educación del sobredotado y el desarrollo del talento para todos. Revista de Psicologia (Lima), 26(1), 25-44.

Reuter, M., Panksepp, J., Schnabel, N., Kellerhoff, N., Kempel, P., \& Hennig, J. (2005). Personality and biological markers of creativity. European Journal of Personality, 19(2), 83-95. doi:10.1002/per.534

Schultz, S. (2009). Twice-exceptional students participating in advanced placement. Saarbrucken, Germany: VDM.

Sligh, A. C., Conners, F. A., \& Roskos-Ewoldsen, B. (2005). Relation of creativity to fluid and crystallized intelligence. The Journal of Creative Behavior, 39(2), 123-136. doi:10.1002/j.2162-6057.2005.tb01254.x

Sternberg, R. J., \& Grigorenko, E. L. (2001). Guilford's structure of intellect model and model of creativity: Contributions and limitations. Creativity Research Journal, 13(3-4), 309-316. doi:10.1207/s15326934crj1334_08

Sternberg, R. J., \& O'Hara, L. A. (1999). Creativity and intelligence. In R. J. Sternberg (Ed.), Handbook of creativity (pp. 251-272). Cambridge, MA: Cambridge University Press.

Tafti, M. A., Hameedy, M. A., \& Baghal, N. M. (2009). Dyslexia, a deficit or a difference: Comparing the creativity and memory skills of dyslexic and nondyslexic students in Iran. Social Behavior and Personality, 37(8), 1009-1016. doi:10.2224/ sbp.2009.37.8.1009

Torrance, E. P. (1962). Guiding creative talent. Englewood Cliffs, NJ: Prentice-Hall.

Torrance, E. P. (1965). Rewarding creative behavior. Englewood Cliffs, NJ: Prentice Hall.

Vernon, P. E. (1964). Creativity and intelligence. Educational Research, 6(3), 163-169. doi:10.1080/0013188640060301

Wechsler, S. M. (2003). DFH III: O desenho da figura humana: Avaliação do desenvolvimento cognitivo infantil (3. ed. ampl. atual.). Campinas, SP: Impressão Digital do Brasil.

Wechsler, S. M. (2008). Estilos de pensar e criar: Impacto nas áreas educacional e profissional. Psicodebate, 7, 207-218.

Wechsler, S. M., Nunes, M. F. O., Schelini, P. W., Ferreira, A. A., \& Pereira, D. A. P. (2010). Criatividade e inteligência: Analisando semelhanças e discrepâncias no desenvolvimento. Estudos de Psicologia (Natal), 15(3), 243-250. doi:10.1590/ s1413-294x2010000300003

West, T. G. (2009). In the mind's eye: Creative visual thinkers, gifted dyslexics, and the rise of visual technologies. New York: Prometheus Books.

Winner, E., von Karolyi, C., Malinsky, D., French, L., Seliger, C., Ross, E., \& Weber, C. (2001). Dyslexia and visual-spatial talents: Compensation vs. deficit model. Brain and Language, 76(2), 81-110. doi:10.1006/brln.2000.2392

Zenasni, F., \& Lubart, T. I. (2008). Emotion-related traits moderate the impact of emotional state on creative performance. Journal of Individual Differences, 29(3), 157-167. doi:10.1027/1614-0001.29.3.157

Recebido: 03/02/2014

$1^{a}$ revisão: $10 / 03 / 2014$

$2^{a}$ revisão: 16/04/2014

Aceite final: 25/04/2014 